\title{
Zur Einführung einer neuen ,Kunstwährung' an den Hochschulen - Die Vergleichs- und Tauschfunktion von ECTS-Punkten
}

\author{
Stefan Kühl
}

\section{$1 \quad$ Einleitung}

Wie lässt sich der überraschende Erfolg der Bologna-Reform erklären? In diesem Artikel wird die Metapher der ECTS-Punkte als eine ,Art von internationaler Währung', als ,Gemeinsame Währung', als ,Bildungswährung' oder als ,Hochschulbildungswährung“ aufgegriffen. Es wird gezeigt, dass die Währungsmetapher nur Sinn macht, weil die ECTSPunkte an den Hochschulen nicht lediglich zu internen Vergleichszwecken gebraucht werden, sondern die akkumulierten Punkte am Ende gegen einen Studienabschluss getauscht werden können. Die Währungsmetapher nutzend, werden hier verschiedene Aspekte des Erwerbs, des Tausches und der Sammlung der Punkte näher analysiert, und es wird gezeigt, auf welche Weise die ,Werthaftigkeit' der Kunstwährung gesichert wird. Die These ist, dass die Sogwirkung der ECTS-Punkte maßgeblich für den (Verbreitungs-)Erfolg der Bologna-Reform verantwortlich ist ${ }^{1}$.

\section{Zum überraschenden Erfolg einer Hochschulreform}

Eigentlich hätte man davon ausgehen können, dass der von den europäischen Bildungsministern in Bologna angestoßene Prozess sicher zum Scheitern verurteilt ist. Die BolognaErklärung hat keinerlei rechtliche Verbindlichkeit für die Unterzeichnerstaaten, weil in den Grundlagenverträgen der Europäischen Union eindeutig geregelt ist, dass die Bildungspolitik einzig und allein in die Kompetenz der jeweiligen Nationalstaaten fällt und Vereinbarungen auf EU-Ebene zur Bildungspolitik keine Verbindlichkeiten für die Unterzeichnenden nach sich ziehen. Alle früheren Initiativen zur Hochschulpolitik der Europäischen Kommission wie z. B. ein Memorandum zum Europäischen Hochschulraum wurden deswegen von den Regierungen der europäischen Staaten argwöhnisch beäugt und dann faktisch ignoriert (zu dem Aspekt des erwarteten Scheiterns ausführlich vgl. Maeße 2010: 19).

Umso erstaunlicher ist, mit welcher Geschwindigkeit sich der Bologna-Prozess durchsetzte - und zwar nicht nur als eine unverbindliche Unterschrift unter ein Abkommen, sondern mit konkreten Auswirkungen. Schon bei der Bologna-Konferenz hatten sich bereits dreißig europäische Staaten auf eine gemeinsame Politik geeinigt. Inzwischen haben sich über vierzig Staaten dem ursprünglich auf Europa beschränkten Bologna-Prozess an-

\footnotetext{
${ }^{1}$ Bei diesem Artikel handelt es sich um eine Vorstudie vor mein Buch „Der Sudoku-Effekt. Hochschulen im Teufelskreis der Bürokratie“ (Transcript Verlag 2012). Die Überlegungen sind in das zweite Kapitel „Kleine Punkte, große Wirkung“ eingegangen.
} 
geschlossen, und selbst Staaten wie Moldawien, Russland oder Kasachstan, die normalerweise nicht unbedingt zu den europäischen Kernstaaten gerechnet werden, würden sich inzwischen zu den Prinzipien eines gemeinsamen europäischen Hochschulraums bekennen. ,Bolognaropa ‘ erstreckt jetzt schon von Tromsø in Norwegen bis Nikosia auf Zypern, von Reykjavik in Island bis Wladiwostok in Russland (Keller 2005: 63).

Wie ist der (Verbreitungs-)Erfolg der Bologna-Reform gerade im Gegensatz zu den vielen vorher gescheiterten europäischen Reforminitiativen zu erklären?

Die These dieses Artikels ist, dass der Verbreitungserfolg - bei allen nationalen Sondersituationen - nur durch die Wirkweise des europäischen Kreditpunktesystems, das ein zentraler Bestandteil der Reform (geworden) ist, erklärt werden kann. ECTS-Punkte sind „ein quantitatives Maß für die Gesamtbelastung des Studierenden“ (KMK 2004: 3). Es handelt sich bei den Punkten keineswegs um eine beschönigende Bezeichnung für Noten: Mit ihnen werden die Zeitstunden gemessen, die ein ,durchschnittlicher Student ${ }^{\text {' }}$ mit der Vorbereitung des Lehrstoffs, der Prüfungsvorbereitung, der Abfassung einer Hausarbeit, der Absolvierung eines Praktikums und der Anfertigung einer Abschlussarbeit verbringt.

Mit den sogenannten European-Credit-Transfer-System-Punkten (ECTS-Punkten) wurde - übrigens zeitlich fast parallel zur Einführung des Euros als gemeinsame europäische Währung - an den Universitäten eine ,Kunstwährung ' verpflichtend eingeführt ${ }^{2}$. Mit der Bezeichnung der ECTS-Punkte als eine „Art von internationaler Währung“ (Erhardt 2000: ix), als „Gemeinsame Währung“ (Adelmann 2009), als „Bildungswährung“ (Roscher 2000: 50) oder als „Hochschulbildungswährung“ (Winter 2009: 22) wurde besonders auf die durch die ECTS-Punkte geschaffene Möglichkeit der Vergleichbarkeit von Studienleistungen abgezielt. Während früher - so das Bild - an den Universitäten eine primitive Sammel- und Tauschwirtschaft herrschte, in der jede Universität ihre eigenen ,Scheine" produzieren konnte, die nur an ihrer eigenen Universität gegen einen Studienabschluss eingetauscht werden konnten, würde jetzt eine einheitliche ,Kunstwährung“ geschaffen, mit der Studienleistungen im Prinzip weltweit verglichen und damit auch transferiert werden könnten ${ }^{3}$.

Die Einführung dieser für die meisten Universitäten neuen Zeitberechnungsform wurde begleitet von der häufig in dramatische Worte gekleideten Verkündigung eines ,Paradigmenwechsels“ von einer ,Lehrendenorientierung، des Studiums zu einer ,Studierendenorientierung'. Während früher, so die Behauptung, die Studiengangsplanung immer von den ,Kontaktzeiten der Lehrenden' mit ihren Studierenden ausgegangen sei, würde mit Einführung der neuen ,Kunstwährung' bei der Studiengangsplanung systematisch von dem „gesamten Zeitaufwand der Studierenden“" aus gedacht werden (vgl. Wagner 2007: 107). Auch wenn diese Verkündigung eines Wechsels von einer ,Lehrendenorientierung zur ,Studierendenorientierung' nur begrenzt logisch erscheint, weil ja die ,Kontaktzeiten der Lehrenden' mit ihren Studierenden immer auch gleichzeitig die ,Kontaktzeiten der Studie-

\footnotetext{
${ }^{2}$ Woher die Metapher der ECTS-Punkte als Währung kommt, ließ sich von mir bisher nicht genau rekonstruieren. Schon relativ früh wird von den ECTS-Punkten als ,eine Art internationale Währung“ (Erhardt 2000: 3) geschrieben. Ich nutze hier den Begriff der ,Kunstwährung', um auf spezifische Merkmale - gerade auch in Abgrenzung zu Geld - hinzuweisen.

${ }^{3}$ Es wird argumentiert, dass zwei soziale Formen - ,Tauschen' und ,Vergleichen ' - in Kunstwährungen (aber natürlich auch bei Geld) zusammenkommen. Auf die Theorie des Tauschens und Vergleichens werde ich immer nur dann eingehen, wenn es für meine Argumentation relevant ist. Die Ausarbeitung des Arguments, weswegen der Begriff der ,Kunstwährung' nicht nur für das Funktionssystem der Wirtschaft, sondern auch für andere Funktionssysteme genutzt wird und welche Unterschiede es macht, ob eine Kunstwährung im Funktionssystem der Wirtschaft oder im Funktionssystem der Erziehung eingesetzt wird, wird von mir an einer anderen Stelle geleistet.
} 
renden mit ihren Lehrenden' sind, liefern die mächtigen Worte des ,Paradigmenwechsels“ und der ,kopernikanischen Wende" doch die notwendige hochschuldidaktische Begleitmusik für eine erstmal dröge daherkommende Einführung einer neuen Verrechnungseinheit.

Durch die ECTS-Punkte solle es also - so jedenfalls die Vorstellung der Bildungsplanung - möglich sein, jede Stunde, die ein Studierender mit seinem Studium verbringt, im Voraus zu kalkulieren. Dabei wird - ohne systematisch empirische Erhebungen über faktisches Studierverhalten in verschiedenen Studiengängen herangezogen zu haben davon ausgegangen, dass der Student ,Otto Normalverbraucher" und die Studentin ,Erika Mustermann“ in Deutschland, Ungarn, Rumänien oder Belgien durchschnittlich im Semester 900 Stunden studieren (30 Leistungspunkte, wobei ein Leistungspunkt für 30 Stunden steht), während davon ausgegangen wird, dass die österreichischen, spanischen und kroatischen Studierenden lediglich 750 Stunden pro Semester mit ihrem Studium verbringen (30 Leistungspunkte pro Semester, wobei ein Leistungspunkt für 25 Stunden steht). Die so errechneten Stunden pro Semester werden dann auf die Stunde genau auf die Anforderungen, die an einen Studierenden mit Unterricht, Unterrichtsvorbereitung, Prüfungsvorbereitung, Prüfung und Praktika in einem Semester gestellt werden, heruntergebrochen ${ }^{4}$.

Im folgenden zweiten Abschnitt wird die Metapher von den ECTS-Punkten als ,internationale Währung،, als ,gemeinsame Währung، oder als ,Hochschulbildungswährung' aufgegriffen, um - im Vergleich zu anderen Kunstwährungen sowohl im Wirtschaftsals auch im Erziehungssystem - zu zeigen, welche Veränderungen durch die verpflichtende Berechnung aller erwarteten Studienleistungen in einer vorgegebenen Zeiteinheit an den Hochschulen bewirkt wurden. Im dritten Abschnitt wird gezeigt, wie über Akkreditierungsagenturen, Qualitätsmanagementstellen und Studiendatenbanken die Kunstwährung gemanagt wird. Aufbauend auf dieser ausführlichen Darstellung der Funktionsweise des Kreditpunktesystems wird dann in Abschnitt vier gezeigt, wie es zu einer räumlichen Ausbreitung, sozialen Ausweitung und sachlichen Ausdehnung der Kunstwährung gekommen ist. Im abschließenden fünften Abschnitt wird kurz zusammengefasst, inwieweit von den ECTS-Punkten eine Sogwirkung für die gesamte Hochschulreform ausgegangen ist.

\section{Die Einführung von ECTS-Punkten als zentralem Element zum Verständnis der Hochschulreform}

Kunstwährungen wie die ECTS-Punkte, die Miles-and-More-Punkte von Fluggesellschaften, die Sammelpunkte beim Kauf eines Schokoladenriegels oder auch die Stempelkarten beim Friseur, mit denen man den elften Haarschnitt umsonst bekommt, basieren immer auf irgendeiner Form von Zählung. Dabei sind die durch mehr oder weniger

\footnotetext{
${ }^{4}$ Die Tatsache, dass man bei aller ECTS-Arithmetik keinerlei Information über das faktische Studierverhalten hat, wird daran deutlich, dass die „Messung und Kontrolle der Arbeitsbelastung von Studierenden“ immer nur als Ziel angeführt wird. So heißt es beispielsweise im Deutschen Nationalbericht der Bologna Follow-up Group Deutschland (2009: 27), dass die „Erhebung der studentischen Arbeitsbelastung ... im Rahmen der Reakkreditierung zunehmend Grundlage der Curriculumsgestaltung“ wird und „bei Veranstaltungen und Informationsangeboten“ immer auf die „Bedeutung der Erhebung und regelmäßigen Überprüfung“ hingewiesen wird. Zu Deutsch: Man hat faktisch keine Ahnung, wie viel Zeit Studierende für die Komponenten ihres jeweiligen Studiums wirklich brauchen.
} 
standardisierte Erhebungsmethoden produzierten Zahlen erst einmal nur quantifizierte Informationen, die sich zu einer spezifischen Beschreibung von Merkmalen eignen: die Anzahl der Studierenden an einer Universität, der verkauften Mittagessen in der Mensa oder die Anzahl der Busse zum Hauptgebäude (vgl. Heintz 2010: 167). So nützlich quantitativ erhobene Informationen auch sein mögen, so gut sie auch mit anderen Zahlen kombiniert werden können und so gut sie sich auch miteinander vergleichen lassen - eine Zahl an sich ist noch lange keine Währung.

$\mathrm{Zu}$ einer ,Kunstwährung' werden diese Zahlen erst, wenn sie mit der Möglichkeit des Tausches ,aufgeladen" werden. Die Sammelpunkte, die man gerade vor großen Sportereignissen beim Kauf eines hochpreisigen Schokoriegels erhält, werden nur deswegen zur Kunstwährung, weil sie gegen einen Fußball, ein T-Shirt der Lieblingsmannschaft oder eine bunte Baseballmütze eingetauscht werden können. Die Smileys - um eine eher primitive Form von Kunstwährung aus dem Erziehungssystem zu nennen -, die man für braves Verhalten vom Grundschullehrer ins Heft gemalt bekommt, lassen sich gegen Aufkleber mit verschiedenen Comic-Motiven oder andere kleine Geschenke tauschen. Miles-and-More-Punkte werden durch das Abfliegen von (nicht notwendigerweise selbst bezahlten) Flügen verdient, elektronisch bei den Fluggesellschaften gespeichert und dann als Kunstwährung gegen Freiflüge, Hotelübernachtungen oder VIP-Tickets für Klassikkonzerte getauscht.

\subsection{Wogegen kann man eine Kunstwährung eintauschen? Tausche Punkte gegen Studienabschluss}

,Kunstwährungen“ unterscheiden sich von einer Geldwährung dadurch, dass ihre Möglichkeiten zum Tausch begrenzt sind. Während Geld im Prinzip zum Erwerb fast jeder Sache und jeder Leistung geeignet ist, können Kunstwährungen immer nur gegen wenige genau definierte Produkte und Leistungen eingetauscht werden. Mit meinen Miles-andMore-Punkten kann ich nicht im Supermarkt einkaufen gehen, mit Hanuta-Sammelpunkten kann man nicht ein Fahrrad, eine Krankenversicherung oder eine Prostituierte bezahlen, und die Smileys kann man noch nicht einmal gegen gute Noten eintauschen, sondern eben nur gegen bunte Aufkleber 5 .

Vor der Einführung der Bologna-Studiengänge gab es an Universitäten - wenn überhaupt - lediglich eine äußerst rudimentäre Form von Kunstwährung. Durch die Studienordnung war genau vorgegeben, welche Leistungsnachweise in Form von besuchten Veranstaltungen oder abgelegten Prüfungen zu erbringen waren. Man ließ sich als Studierende den Besuch eines Seminars und die Abfassung einer Hausarbeit oder den Besuch einer Veranstaltung mit anschließendem Schreiben einer Klausur auf einem Schein bestätigen. Die Studierenden sammelten diese Scheine dann in einem Studienbuch, das sie möglichst nicht verlieren sollten, weil ihre erbrachten Leistungen nur dort dokumentiert waren. Dabei war die Art und Anzahl der erforderlichen Scheine von Studiengang zu Studiengang sehr verschieden. In einigen Studiengängen mussten 17 Scheine erworben

\footnotetext{
${ }^{5}$ Selbstverständlich gibt es auch in einer durch Geld geprägten Gesellschaft Grenzen der Käuflichkeit. ,Geld` mag Männer (oder zunehmend auch Frauen), sexy` machen, und es mag sehr wohl zum Liebesspiel gehören, dass einer der beiden Geschlechtspartner durch das Bezahlen von Essen, Kleidung oder Wohnung seine Großzügigkeit signalisiert, aber sowohl der Mann als auch die Frau achten normalerweise darauf, dass der Geschlechtsverkehr nicht als Tausch einer sexuellen Leistung gegen Geld missverstanden werden kann.
} 
werden, in anderen reichten fünf Scheine mit entsprechend nachgewiesenen Leistungen aus $^{6}$. Aber selbst wenn man alle durch die Studienordnung vorgeschriebenen Scheine zusammen hatte, konnte man diese nicht einfach gegen einen Abschluss in Physik, Sportwissenschaft oder Philosophie eintauschen, sondern nur gegen die Berechtigung, die Abschlussprüfungen ablegen zu dürfen. Den mit einer entsprechenden Note ausgestatteten Abschluss erhielt man dann erst im Tausch gegen die bestandenen Prüfungen.

Erst durch die Einführung von ECTS-Punkten wurde letztlich eine funktionierende ,Kunstwährung' im Bereich der Erziehung geschaffen. Studierende können durch die Kunstwährung an verschiedenen Universitäten ihre ECTS-Punkte sammeln, diese über einen längeren Zeitraum in ihren sogenannten Transcripten speichern und an ihre Heimatuniversität transferieren lassen. Wenn sie genug zu ihrem Studienfach passende ECTSPunkte gesammelt haben, können sie diese dann an ihrer Heimatuniversität gegen einen Studienabschluss eintauschen.

In einem zentralen Aspekt unterscheidet sich die Kunstwährung, ECTS‘ von einer Reihe von anderen Kunstwährungen und besonders von Geldwährungen: Der Erwerb, Besitz und Tausch der Währung ist an eine konkrete Person gebunden. Man kann einen ECTS-Punkt nicht einfach an eine Kommilitonin oder einen Kommilitonen weiterverschenken. Der Punkt hat nur für die Person, die ihn erworben hat, einen Tauschwert. Wir kennen diese Bindung einer Berechnungseinheit an Personen auch von anderen Kunstwährungen. Die Miles-and-More-Punkte in den Vielfliegerprogrammen erwirbt man personenbezogen. Man kann diese Punkte - jedenfalls in der einfachen Form der Vielfliegerprogramme - nicht einfach weiterverschenken, weitertauschen oder gar weiterverkaufen?

Gerade diese Bindung an Personen schränkt die Möglichkeit des Tausches im Vergleich zu anderen Kunstwährungen wie Duplo-Sammelpunkte oder Friseur-Sammelpunkte stark ein. Während Duplo-Sammelpunkte oder Friseur-Sammelpunkte - jedenfalls im Prinzip - immer von Person zu Person weitergetauscht werden können und so die Tauschwährung mit einer hohen Geschwindigkeit umgeschlagen werden kann, können ECTS-Punkte nur von der Person, die sie erworben hat, ein einziges Mal bei der Universität gegen einen Studienabschluss eingetauscht werden. Sind die ECTS-Punkte von Studierenden erst einmal gegen den Studienabschluss eingetauscht worden, dann werden sie letztlich in den Tiefen der EDV-Systeme der Universität vergraben ${ }^{8}$.

\footnotetext{
${ }^{6}$ In der durch die Studentenrevolten losgetretenen Reform-Euphorie der siebziger Jahre des zwanzigsten Jahrhunderts gab es sogar einige Studiengänge, die ihre Studierenden komplett davon entlasteten, während ihres Studiums irgendwelche Scheine zu erwerben.

${ }^{7}$ Die Experimente mit der Weiterentwicklung der Vielfliegerprogramme bestehen gerade darin, diese Personenbezogenheit aufzulösen. Während man in der weiterentwickelten Form der Vielfliegerprogramme die Bonusmeilen teilweise weiterverschenken oder weitertauschen kann, bleiben die Statusmeilen, mit denen man Loungezugänge, Sitzplatzreservierungen oder Buchungsprivilegien erwirbt, personengebunden.

${ }^{8}$ Die Universität, die die ECTS-Punkte gegen einen Studienabschluss eingetauscht hat, kann diese ECTS-Punkte nicht erneut weitertauschen. Sie werden nicht einfach - bei entsprechenden Leistungen - an andere Studierende neu vergeben, sie können von der Universität nicht als Tauschgut mit anderen Universitäten genutzt werden, und sie können nicht (jedenfalls noch nicht) bei staatlichen Stellen z. B. gegen Geldmittel eingetauscht werden.
} 


\subsection{Wie wird die Kunstwährung erworben? Die Renaissance der Arbeitswerttheorie an den Universitäten}

Für das Verständnis der Wirkweise einer ,Kunstwährung' ist es zentral, zu begreifen, wie sie erworben werden kann. Die Sammelpunkte bekommt man parallel zum Kauf eines Duplos oder Hanutas. Die Bonuspunkte eines Vielfliegerprogramms erwirbt man durch das Abfliegen von häufig durch andere bezahlten Flügen, durch die Nutzung einer Kreditkarte, das Ausfüllen eines Fragebogens oder auch nur durch die Bereitschaft, sich in kurzen Zeitabständen mit Werbeangeboten bombardieren zu lassen. Bei dem ECTS-System erwerben Studierende Punkte, die erst einmal nur die Zeit repräsentieren, die - nach Einschätzung der Universität - für die Vor- und Nachbereitung eines Seminars, für den Besuch einer Vorlesung oder das Abfassen einer Hausarbeit - nötig ist und tauschen diese Zeiteinheiten dann gegen einen Studienabschluss. Eine Umstellung, die die Kalkulationsweise an den Universitäten grundlegend verändert.

Vor der Einführung des ECTS-Systems bestand das Interesse der Universitäten lediglich darin, sicherzustellen, dass Studierende eine vorgeschriebene Anzahl von Vorlesungen, Seminaren, Übungen, Hausarbeiten, Klausuren und mündlichen Prüfungen erfolgreich absolvierten. Gezählt wurde lediglich in der vermutlich für Außenstehende kompliziert klingenden Einheit der ,Semesterwochenstunden' - also den Stunden, die ein Studierender während des Semesters pro Woche in Veranstaltungen zubringt ${ }^{9}$. Welche Zeit die Studierenden brauchten, um die erforderlichen Leistungsnachweise für diese Veranstaltungen zu erbringen, interessierte letztlich niemanden. Eine brillante Hausarbeit, die ein Studierender in 40 Stunden erbracht hat, galt mehr als eine schäbige Ausarbeitung, für die ein Studierender 200 Stunden gebraucht hat ${ }^{10}$. Ein bisschen erinnert dieses inzwischen weitgehend ausgesetzte System an die in der Marktwirtschaft praktizierten Kalkulationsformen. Einem Käufer ist es in der Marktwirtschaft letztlich egal, ob für die Produktion eines Wasserkochers insgesamt 25 oder 70 Arbeitsstunden nötig gewesen sind, sein Kaufverhalten wird lediglich durch den Preis und die Qualität des Produktes beeinflusst. Wenn es einem Anbieter gelingt, einen qualitätsmäßig hochwertigen Wasserkocher zu einem günstigen Preis in 25 Stunden statt in 70 Stunden zu produzieren - umso besser.

Mit der Einführung des ECTS-Systems und der damit einhergehenden Verrechnung von Leistungen in Zeitstunden wurde jetzt - für Beteiligte, die ihr Studium in den 1970er Jahren absolviert haben, vermutlich bewusst, für die meisten Beteiligten jedoch unbewusst - eine alte volkswirtschaftliche Idee, die Arbeitswerttheorie, in die Praxis umgesetzt ${ }^{11}$.

\footnotetext{
${ }^{9}$ Dieses alte Modell der Semesterwochenstunden ähnelte dem Anfang des 20. Jahrhunderts eingeführten CreditSystem in den USA, in dem ein Credit etwa ,einer Stunde wissenschaftlicher Arbeit im Seminarraum, in der Bibliothek oder im Labor entsprechen sollte“, das Studium zu Hause, unmittelbares Arbeiten mit Dozenten oder anderen Studierenden oder Prüfungsvorbereitungen jedoch nicht abbildete (siehe für einen kurzen deutschsprachigen Überblick über den Charakter der sogenannten „Carnegie Unit“ Altbach 2000: 83).

${ }^{10}$ Sicherlich: Auch vor der Einführung der ECTS-Punkte haben Lehrende kalkuliert, wie viel Zeit Studierende für die Vor- und Nachbereitung von Veranstaltungen, für die Abfassung von Hausarbeiten oder das Lernen für Prüfungen brauchen. Aber diese Kalkulation fand Pi mal Daumen statt, die Schätzungen wurden nirgends schriftlich niedergelegt und mussten nicht gegenüber Außenstehenden gerechtfertigt werden. Am Ende interessierte lediglich, dass Studierende eine genau definierte Anzahl von Leistungen erbringen.

${ }^{11}$ Inwiefern bei der Entwicklung des European Credit Transfer System in den 1980er Jahren systematisch Überlegungen aus der Arbeitswerttheorie herangezogen wurden, müsste wissenschaftshistorisch noch näher untersucht werden. Da gerade die in jener Zeit aktiven Bildungsplaner und Bildungswissenschaftler häufig in den frühen 1970er Jahren durch intensive Studien des Kapitals von Marx erzogen - oder sollte man sagen: sozialisiert -
} 
Nach der auf Karl Marx - und davor besonders auf den Nationalökonomen David Ricardo - zurückgehenden volkswirtschaftlichen Arbeitswerttheorie wird der Wert einer Ware nicht durch die auf dem Markt erzielbaren Preise bestimmt, sondern einzig und allein durch die Arbeitszeit, die zu ihrer Herstellung notwendig ist ${ }^{12}$. Mit Karl Marx lässt sich die Idee hinter den ECTS so formulieren, dass der „Wert einer Ware“ - hier also einer Studienleistung - durch „die zu ihrer Herstellung erforderliche Arbeitsmenge“ bestimmt wird. Deswegen müsse der „Wert der Arbeit“ - bei Marx der Arbeitslohn, beim ECTS die Bescheinigung von Studienleistungen - ,gleichfalls durch die Arbeitsmenge bestimmt werden, die zu seiner Herstellung erforderlich ist" (vgl. Marx 1953: 487) ${ }^{13}$.

Genauso wie Karl Marx sich bewusst war, dass sich nicht für jedes Produkt und jede Dienstleistung exakt sagen ließ, wie viel Zeit jeder einzelne Mensch für dessen bzw. deren Herstellung braucht, sind sich auch die Erfinder des European Credit Transfer and Accumulation System darüber im Klaren, dass sich nicht für jeden Studierenden genau sagen lässt, wie viel Zeit er für das Lesen eines Textes, die Nachbereitung einer Vorlesung oder das Abfassen einer Hausarbeit braucht. Genauso wie in der Marx'schen Arbeitswerttheorie bei der Kalkulation von Zeitanforderungen also von einem ,Durchschnittsarbeiter ' ausgegangen wird, wird im Kreditpunkte-System von der Arbeitsbelastung für einen Studierenden ausgegangen, der entsprechend der ,Zielgruppendefinition ein ,normales" Profil" aufweist (vgl. Gehmlich 2000: 61). Die Berechnungsgrundlage in der Arbeitswerttheorie ist also immer nur die durchschnittliche Arbeitszeit.

Bei der Entwicklung der Arbeitswerttheorie als Instrument zur Steuerung innerhalb und zwischen Organisationen war man sich selbstverständlich bewusst, dass man eine in der vorgesehenen Durchschnittsarbeitszeit erbrachte Leistung unterschiedlich gut erbringen kann. Während in der sozialistischen Planwirtschaft die während der durchschnittlichen Arbeitszeit erbrachte hohe Qualität nur mit einem Lob für eine ,Planübererfüllung“ oder bestenfalls mit Auszeichnungen wie ,Held der Arbeit', ,Karl-Liebknecht-Medaillen“ oder ,Ehrennadeln‘ belohnt wurde, steht bei der Kunstwährung ECTS das aus dem alten System der Leistungsbewertung und Leistungsmotivation übernommene Konzept der Noten zur Verfügung, um zu markieren, wie gut oder schlecht die in der Durchschnittsarbeitszeit erbrachten Leistungen eines Studierenden gewesen sind.

Die Marx'sche Arbeitswerttheorie hat sich praktisch nur in wenigen Feldern des Wirtschaftssystems durchsetzen können. In der staatssozialistischen Planwirtschaft wird basierend auf der Marx'schen Arbeitswerttheorie davon ausgegangen, dass der Wert der Arbeitsstunde eines Bergbauern, eines Straßenbauingenieurs und eines Polizisten gleich ist und dementsprechend auch gleich entlohnt werden sollte. In Tauschringen (lokale Zusammenschlüsse zum direkten Tausch von Gütern und Dienstleistungen) richten sich die Preise

wurden, kann man vermuten, dass sich in Akten der Europäischen Union vereinzelt Referenzen auf die Arbeitswerttheorie finden lassen müssten.

${ }^{12}$ Interessanterweise ist den meisten ,Entwicklern“ des ECTS-Systems im Rahmen des Bologna-Prozesses vermutlich wegen fehlender Grundkenntnis der politischen Ökonomie - diese Parallelität zwischen dem ECTSSystem und der Arbeitswerttheorie nicht aufgefallen. Soziologen, Wirtschaftswissenschaftler und Philosophen, die im Rahmen ihres häufig lange zurückliegenden Studiums noch das Kapital gelesen haben, stolpern fast automatisch darüber, dass mit dem ECTS-System der Grundgedanke der Marx'schen Arbeitswerttheorie aufgegriffen wird (vgl. nur beispielsweise die Hinweise bei Liessmann 2008).

${ }^{13}$ Wörtlich - und ohne Einflechtungen von Referenzen auf das ECTS-System - heißt es bei Marx: „Wenn der Wert einer Ware bestimmt wird durch die zu ihrer Herstellung erforderliche Arbeitsmenge, so folgt daraus, dass der Wert der Arbeit, d.h. der Arbeitslohn, gleichfalls durch die Arbeitsmenge bestimmt wird, die zu seiner Herstellung erforderlich ist" (Marx 1953: Seite?). 
für so unterschiedliche Waren wie eine Massage, eine selbst getöpferte Teekanne und eine Existenzgründerberatung nicht nach Angebot und Nachfrage, sondern nach der Zeit, die für deren Herstellung notwendig ist. In den durch die Marktwirtschaft geprägten Denkwelten hat sich die Arbeitswerttheorie jedoch nicht etablieren können. Es würde - bei aller oberflächlichen Plausibilität der Arbeitswerttheorie - Irritationen auslösen, wenn ein Friseur mit Verweis auf die gleiche Arbeitszeit den gleichen Stundenlohn fordern würde wie ein Topmodel oder eine Universitätsprofessorin. Aber die ECTS-Punkte sind ein Beispiel dafür, dass sich eine von vielen Ökonomen als Erklärung für volkswirtschaftliche Prozesse für ,tot' erklärte Theorie, die sich als Grundlage für die komplexe Steuerung von Wirtschaftsprozessen (jedenfalls bisher) nicht global durchsetzen konnte, sehr wohl zur Steuerung interner Prozesse in Organisationen oder auch zur Steuerung der Austauschprozesse zwischen Organisationen außerhalb der Wirtschaft verwenden lässt.

\subsection{In welchen Einheiten werden Leistungen erworben und getauscht? Module als Container für die neue Kunstwährung}

Kunstwährungen kann man auf unterschiedliche Art und Weise stückeln. Der Normalfall ist sicher, dass Kunstwährungen in einer beliebigen Einheit des Dezimalsystems erworben, kumuliert, gespeichert und getauscht werden können. Die Miles-and-More-, Hanuta- oder Smileypunkte werden beispielsweise in unterschiedlichen Einheiten des Dezimalsystems erworben, sie lassen sich aber zur Vorbereitung des Tausches beliebig miteinander addieren. In diesem Aspekt unterscheiden sie sich nicht von einer Geldwährung, bei der man ja auch selbstverständlich davon ausgeht, dass man alle Euro-Stücke und -Scheine beliebig miteinander kombinieren kann, unabhängig davon, in welchen Größenordnungen man diese ursprünglich erworben oder gespeichert hat.

Bei Kunstwährungen kann man aber auch festlegen, dass die Währungen erst dann einen Wert bekommen sollen, wenn sie in vorher definierten Einheiten zusammengefasst wurden. Man kann beispielsweise den Erwerb eines Hauptpreises bei Schokoladensammelpunkten daran binden, dass der Sammler jeweils 10 Punkte für Hanutas und 10 für Duplos ins Sammelalbum geklebt hat. Man kann theoretisch die Vergabe der Miles-andMore-Prämien daran binden, dass eine genau definierte Anzahl von Meilen für das Abfliegen von Strecken, für die Nutzung von Kreditkarten und für das Anmieten von Leihwagen erworben wurde. Die Kunstwährung ECTS wurde in eine genauso eigenartige Form gebracht. Ein Studierender kann nicht einfach nur einzelne ECTS-Punkte sammeln, indem er nachweist, dass er 30 Stunden mit irgendetwas Universitärem verbracht hat. Der Erwerb von ECTS-Punkten kann - so die Logik - nur im Rahmen von vorgeschriebenen Veranstaltungen und Prüfungen erfolgen, die wiederum in genau definierte Module zusammengefasst sind und am Ende abgeprüft werden können.

Sicherlich: Auch vor Bologna hat eine solche Form der Zusammenfassung von Leistungen existiert. Man konnte ohne Probleme die Vergabe eines Scheins daran binden, dass eine Studentin zwei auf einander aufbauende Vorlesungen besuchte, eine Übung absolvierte und am Ende eine Hausarbeit schrieb. Wenn die Studentin eine dieser Leistungen nicht erbrachte, dann konnte ein Lehrender ihr den Schein verweigern. Einen Wert als Eintauschgut (für die Zulassung zur Abschlussprüfung) hatte eine einzelne Veranstaltung also nur dann, wenn gleichzeitig auch die anderen verlangten Veranstaltungen und Prüfungen wenigstens auf dem Papier belegt wurden. Diese verpflichtende Zusammenfassung von unterschiedlichen Leistungen schien aus didaktischen Gründen Sinn 
zu machen. Lehrende konnten sich überlegen, welche Veranstaltungen zusammenpassen, wie viel Zeit die Studierenden für die Vorbereitung und Nachbereitung der thematisch zusammenhängenden Veranstaltungen brauchen und mit welchen Prüfungsformen diese abgeschlossen werden sollten. Über die Bindung der Scheinvergabe an die Absolvierung aller dieser Leistungen konnte diese im didaktischen Sinne ersonnene Kombipackung den Studierenden aufoktroyiert werden.

Durch die Einführung der Kunstwährung ECTS wird dieses System jedoch radikalisiert. Das Problem bei der Einführung von ,ECTS-Punkten“ als neue Kunstwährung ist, dass man diese nicht einfach nur für das Eintragen in Anwesenheitslisten in Vorlesungen, das Absitzen in Seminaren, das Nachweisen von am Schreibtisch verbrachten Lesezeiten oder das Abfassen von Papieren vergeben kann. Schließlich geht es in Universitäten - so die Logik auch nach der Bologna-Reform - nicht vorrangig um den Nachweis von Anwesenheiten, sondern um den Nachweis des Erlernten, Reflektierten und Angewendeten. Deswegen muss, so jedenfalls die Vorstellung der Bologna-Vordenker, noch einmal abgeprüft werden, ob die in ECTS gemessene Zeit des Studierenden auch wirklich zum Wissenserwerb geführt hat. Über schriftliche Klausuren, Multiple-ChoiceKlausuren, Referate, Arbeitsberichte, mündliche Prüfungen oder Hausarbeiten müsse, so die an den meisten Universitäten dominierende Vorstellung, von den Studierenden bewiesen werden, dass die mit dem Studium verbrachte Zeit ,Effekte' hat. Nur für einen solchen durch Prüfungen zertifizierten Wissenserwerb dürften dann letztlich ECTS-Punkte vergeben werden.

Aus nachvollziehbaren Gründen ist es jedoch kompliziert, den mit jedem einzelnen ECTS-Punkt verbundenen Wissenserwerb abzuprüfen. Bei 180 Leistungspunkten, die man für das Eintauschen gegen einen Studienabschluss in einem Bachelorprogramm braucht, würde das in drei Jahren 180 Einzelprüfungen bedeuten. Aber auch die Abprüfung jeder mit 2, 3 oder 4 Leistungspunkten bemessenen Übung, Vorlesung oder Seminarveranstaltung würde bei einem Bachelor zu 30 bis 50 Einzelprüfungen in drei Jahren führen. In einigen Universitäten mag das unter Bologna-Bedingungen inzwischen Realität geworden sein, aber an den meisten Universitäten wird dies - gerade auch aufgrund der Belastung für das korrigierende Lehrpersonal - als nicht machbar eingeschätzt.

Allein durch die Verrechnung aller Studienleistungen in ,ECTS-Punkten“ und die Konditionierung der Vergabe von ECTS-Punkten an eine bestandene Prüfung ist eine Sogwirkung entstanden, und zwar in der Weise, dass dies die Einführung von Modulen nahelegte, in denen thematisch ähnliche Vorlesungen, Seminare und Übungen zusammengefügt werden und das dort zu vermittelnde Wissen durch eine einzige Prüfung abgenommen werden kann. Diese Module müssen, so die Vorgabe in den meisten Ländern, mit Inhalten und Qualifikationszielen, mit Lehrformen, mit Voraussetzungen für die Teilnahme, mit Verwendbarkeit des Moduls und mit den Voraussetzungen für die Vergabe von Leistungspunkten beschrieben werden. Es muss also bis ins Detail festgelegt werden, welche Vorlesungen, welche Seminare und welche Übungen im Rahmen des Moduls belegt werden müssen, welche Klausuren zu schreiben sind, welche Essays und Hausarbeiten abzufassen sind und wie viel Zeit die Studierenden für die Vor- und Nachbereitung von Sitzungen verbringen sollen. Der Wert der Kunstwährung ECTS zeigt sich also erst, wenn das Lernpensum in Module ${ }^{14}$ gegossen ist.

\footnotetext{
${ }^{14}$ Ich orientiere mich hier am deutschen Modell. Siehe für die deutsche Konzeption der „Modularisierung“ beispielsweise BLK (2002: 4) und KMK (2004: 2). Es gibt also keinen kausalen Nexus zwischen der Einführung
} 
Auch wenn die ECTS-Punkte der Einführung von Modulen den Weg bereitet haben eine Tendenz, die ohnehin kaum zu vermeiden war, darf nicht übersehen werden: Hinter der Modularisierung verbirgt sich ein seit längerer Zeit propagiertes hochschuldidaktisches Konzept. Schon in den Reformdiskursen nach dem Zweiten Weltkrieg hat es immer wieder die Forderung gegeben, unterschiedliche Veranstaltungstypen und Lernformen in themaisch orientierte Module zusammenzufassen (siehe nur die durch die Studentenbewegung in den späten 1968er Jahren inspirierten Überlegungen von Weizsäcker 1970) ${ }^{15}$. Aber während der Modulgedanke in der Nachkriegszeit lediglich ein Gedankenspiel war, das in vielen Ländern höchstens in Pilotprojekten von Reformuniversitäten einmal ausprobiert wurde, hat die Einführung der Kunstwährung ECTS dem Modularisierungsgedanken europaweit den entscheidenden Schub versetzt.

Wie wird die neu geschaffene Kunstwährung an den Universitäten gemanagt?

\section{Die Rolle von Akkreditierung, Qualitätssicherung und Evaluation beim Management der Kunstwährung}

Wenn man von außen auf das neu eingeführte System der Bildungswährung schaut, dann ist ein ECTS-Punkt ist erst einmal nur eine Zahl, die in Kombination mit dem Namen eines Studenten oder einer Studentin auf einer Liste, in einer Excel-Datei einer Fakultät oder einer Datenbank einer Universität abgelegt wird. Die reine Tatsache, dass hinter dem Namen eines Studenten oder einer Studentin ein ECTS-Punkt vermerkt wird, bedeutet noch lange nicht, dass dieser Punkt auch kumuliert, gespeichert, transferiert und am Ende gegen einen Abschluss eingetauscht werden kann.

Für die Analyse einer Kunstwährung ist jetzt interessant, wie eine Objektivierung und Absicherung dieser Zahl vonstattengeht - ein Prozess, der in der Forschung über die soziale Konstruktion von Zahlen mit Begriffen wie ,enactment“ (Radcliffe 1999), „upkeying“ (Vollmer 2006) oder „micro-production of macro-order“ (Pentland 1993) bezeichnet wird. Schließlich muss, damit Studierende sich auf das Sammeln von ,ECTS-Punkten“ einlassen, diese ,Kunstwährung' durch verschiedene Verfahren so abgesichert werden, dass die an einer Universität gesammelten ECTS-Punkte beim Vergleich, bei der Verrechnung und beim Tausch gegen einen Studienabschluss ähnlich unstrittig erscheinen wie die Sekunden bei der Zeitmessung eines Hundertmeterlaufs, der Dollar, mit dem man ein Auto mietet oder die Kilogramme oder Pfunde, mit denen man seine Gewichtszu- und -abnahme beobachten kann (vgl. dazu Heintz 2007: 75; Heintz 2010: 169) ${ }^{16}$. Im Rahmen des Bo-

einer Kunstwährung an den Hochschulen und der Modularisierung. Die „Logik eines Credit-Systems“, so Stefanie Schwarz und Meike Rehburg (2003: 148), erfordert keineswegs „unbedingt eine Modularisierung eines Studiums“. Interessant ist jedoch, dass es in den meisten Ländern des europäischen Hochschulraumes eine Tendenz zur Einführung von Modulen gibt.

${ }^{15}$ Auf die historische Entstehung des Modulgedankens kann an dieser Stelle nicht eingegangen werden. Es wäre jedoch bildungshistorisch interessant, die Entwicklung dieses didaktischen Konzeptes näher zu untersuchen. Besonders in Betracht gezogen werden müssten die schon vor dem Zweiten Weltkrieg in den USA zu beobachtenden Tendenzen zur Modularisierung von Studiengängen.

${ }^{16}$ Wir wissen aus der soziologischen Forschung, dass die Validität von Zahlen in sozialen Zusammenhängen unterschiedlich stark abgesichert wird. Es gibt allgemein akzeptierte mathematische Formeln wie das berühmte „,2 $+2=4$ ", deren Gültigkeit nur von Grundschülern in der ersten Klasse oder besonders raffinierten Philosophen bezweifelt wird (vgl. Bloor 1994). Es gibt die Anzahl von verlegten Wasserleitungen in einer Stadt, über deren konkrete Anzahl unterschiedlichste Meinungen existieren mögen, deren Bestand aber im Rahmen eines Projektes 
logna-Prozesses wurden mehrere Institutionen geschaffen, die diese Absicherung zu garantieren versuchen.

\subsection{Die Rolle von Ministerien, Akkreditierungsagenturen und Universitäten als ,Währungshüter "}

Wie bei jeder Währung, muss auch bei den Leistungspunkten verhindert werden, dass die Tauscheinheit einfach von jedem selbst hergestellt wird. Dabei scheint das Risiko nicht so sehr darin zu bestehen, dass sich jeder Studierende in mühsamer Heimarbeit seine ECTSPunkte selbst ,bastelt', sondern eine Gefahr wird vielmehr darin gesehen, dass Billiganbieter ihre eigenen ECTS-Punkte auf den Markt bringen. Schließlich braucht es ja lediglich eine Einrichtung, die Studierenden - gegen entsprechende monetäre Entlohnung Leistungspunkte ausstellt, ohne dass für das Erreichen dieser Leistungspunkte ein entsprechender Aufwand erbracht werden musste.

Die Rolle des Hüters einer Bildungswährung kann von verschiedenen Institutionen übernommen werden. Das am längsten erprobte Verfahren besteht darin, dass der Staat jeden Studiengang genehmigt und die Studierenden automatisch in diesen mit einem staatlichen Gütesiegel ausgestatteten Studiengängen ECTS-Punkte erwerben können. Eine andere mit der Etablierung des europäischen Hochschulraumes geschaffene Möglichkeit besteht darin, die Genehmigung von Studiengängen an halbstaatliche oder gar private Akkreditierungsagenturen auszulagern. ECTS-Punkte können in dem Fall nur dann zwischen Universitäten getauscht und am Ende gegen einen Studienabschluss eingelöst werden, wenn sie im Rahmen eines offiziell akkreditierten Studiengangs erworben wurden. Man kann aber auch - so die dritte Möglichkeit - die Genehmigung der Studiengänge in die Hand von Universitäten geben, darauf vertrauend, dass gerade die staatlichen Universitäten schon kein Schindluder mit der Vergabe von ECTS-Punkten treiben werden.

Diese Formen der Legitimierung von Studienleistungen hat es schon vor Bologna gegeben, schließlich musste auch damals schon die Akzeptanz der Studienabschlüsse außerhalb der Universitäten sichergestellt werden. Neu mit der Einführung der Kunstwährung ist jedoch, dass ab jetzt - jedenfalls im Prinzip - für jeden dieser ECTS-Punkte überprüft werden muss, ob er in der vorgesehenen Zeit erworben werden kann, wie dieser Erwerb mit dem Erwerb anderer ECTS-Punkte zusammenpasst und in welcher Form der Erwerb dieser ECTS-Punkte abgeprüft werden kann. Auch wenn diese Prüfung durch Ministerien, Akkreditierungsagenturen oder Universitäten in der Praxis selten auf der Ebene einzelner Punkte stattfindet, muss doch wenigstens durch eine staatliche Zertifizierung, offizielle Akkreditierung oder universitätsinterne Absegnung die Fiktion produziert werden, dass ein ECTS-Punkt den offiziellen Anforderungen entspricht ansonsten gäbe es ja keine Berechtigung, diese ECTS-Punkte mit anderen Universitäten zu verrechnen.

objektiviert werden kann (vgl. Rottenburg 2001). Und es gibt Indikatoren wie den Korruptionsindex von Transparency International, bei dem die ,soziale Konstruiertheit“ selbst in den Massenmedien diskutiert wird (vgl. Booysen 2002). 


\subsection{Qualitätsmanagement zur Verhinderung von Währungsinflation und Währungsdeflation}

Aber die Genehmigung eines Studiengangs durch Bildungsministerien, Akkreditierungsagenturen oder Universitäten allein reicht nicht aus. Schließlich wird dadurch lediglich sichergestellt, dass die Planung eines Studiengangs inklusive der Berechnung der Arbeitsstunden der Studierenden den Bologna-Kriterien entspricht. Ob sich im alltäglichen Betrieb beispielsweise eine zu laxe Vergabe von ECTS-Punkten einschleicht, die Arbeitsbelastung in einzelnen Modulen durch überdimensionierte Anforderungen eines Lehrenden überdimensional anwächst oder der Erwerb von ECTS-Punkten in der Praxis nicht ausreichend durch Prüfungen abgesichert wird, kann nur durch eigene Sicherungsmechanismen der Universität kontrolliert werden.

Die Etablierung einer Kunstwährung an den Universitäten wurde deswegen mit einer Diskussion über Mechanismen des ,Qualitätsmanagements ${ }^{6}$ an Universitäten verquickt. Diese Diskussion darüber, wie Instrumente des Qualitätsmanagements aus Unternehmen auf Universitäten übertragen werden können, hatte schon vor der Bologna-Reform eingesetzt. Es gab häufig zuerst nur theoretische Überlegungen, wie man die Hochschulen mit den DIN-Qualitätsnormen 9000 ff. zertifizieren könnte, wie ein Total-Quality-Management an den Universitäten aussehen könnte und in welcher Form sogenanntes Benchmarking - Vergleiche zwischen mehreren Universitäten - zu einer Verbesserung der Qualität der Lehre beitragen könnte. Aber durch die Bologna-Reform bekam die Qualitätsdiskussion eine ganz neue Dynamik. Emphatisch wurde von den Bildungsministern betont, „dass die Qualität der Hochschulbildung der Dreh- und Angelpunkt für die Schaffung des Europäischen Hochschulraums ist" und dass jede einzelne Universität entsprechende Maßnahmen treffen müsse, um diese Qualität sicherzustellen (Berliner Erklärung 2003: 3).

Mit der Einrichtung von universitätsweiten Qualitätsmanagementsystemen, eigenen Prorektoraten für Qualitätssicherung und speziellen Abteilungen für Qualitätsentwicklung sah man sich aber schnell mit dem bekannten Problem konfrontiert, dass sich der eigentliche universitäre Kernprozess der Wissensvermittlung in den Seminaren, Vorlesungen und Übungen nur schwer durch die in Unternehmen erprobten Instrumente der Qualitätssicherung erfassen lässt. Es ist schwierig, die ,Ergebnisqualität' von Studierenden z. B. in Form von Reflektionsfähigkeit, Kritikfähigkeit oder Wissensaneignungsfähigkeit zu messen (vgl. dazu Pasternack 2000: 41). Aber auch die ,Prozessqualität', also die Qualität von Lehrveranstaltungen, lässt sich nur schwer evaluieren, weil die Zufriedenheit oder Unzufriedenheit von Studierenden mit einer Lehrveranstaltung häufig nichts über die Qualität einer Veranstaltung aussagt. Schließlich werden dabei häufig nur ,Happy-Points“ vergeben, die nichts darüber aussagen, ob die Studierenden wirklich etwas gelernt haben. Aber auch die ,Strukturqualität', also die Art und Weise, wie Lehrveranstaltungen abgehalten werden, lässt sich kaum bestimmen, schließlich streiten sich selbst Lehrende darüber, ob der Einsatz von PowerPoint-Folien in Vorlesungen jetzt als Zeichen der Beherrschung neuester didaktischer Methoden gewertet werden kann oder nur Ausdruck der rhetorischen Unfähigkeit des Dozenten ist.

Das Qualitätsmanagement an Universitäten kann sich deswegen - abgesehen von der Gewährleistung, dass überhaupt irgendeine Form von Evaluation in den Lehrveranstaltungen stattfindet - vorrangig nur auf die Einhaltung formaler Standards konzentrieren. Somit sind durch die Europäische Union finanzierte Handreichungen auch voller Aussagen, dass durch ein universitätsweites Qualitätsmanagement sichergestellt werden muss, dass der Zeitaufwand für jeden ECTS-Punkt realistisch eingeschätzt wird, dass der benötigte 
Zeitaufwand der Studierenden regelmäßig überprüft wird und dass bei Diskrepanzen die kalkulierten ECTS-Punkte, die Lernziele oder die Lernmethoden angepasst werden (European Communities 2009: 18). Es wird zur , guten Praxis‘ erklärt, dass alle Module eines Studiengangs mit ,geeigneten Lernzielen` beschrieben werden und für jede Komponente eines Moduls klare Informationen über die zu vergebenden Kreditpunkte verfügbar sind (European Communities 2009: 26).

Mit diesen Verfahren wird zwar nicht sichergestellt, dass die Studierenden während ihres Studiums etwas lernen, aber es kann überzeugend nach außen signalisiert werden, dass die ECTS-Punkte sich zur Verrechnung in diesem Studiengang oder mit anderen Studiengängen eignen.

\subsection{Die Einführung von Datenbanken zur Speicherung, zur Kumulierung und zum Tausch von Leistungspunkten}

Ein weiteres Problem betrifft die Gewähr, dass die ECTS-Punkte verlässlich auf Studierende zugerechnet werden können. Auf den ersten Blick wäre das effizienteste Verfahren für die Sammlung und den Tausch der ECTS-Punkte, den Studierenden Leistungspunkte in Form von Münzen und Scheinen auszuhändigen. Für jede Übung, jede Klausur oder jede Vorlesung erhielte ein Studierender von seinen Lehrern eine Münze im Wert von einem, zwei oder drei Leistungspunkten. Für größere Arbeitspakete wie Praktika, Seminare oder Hausarbeiten bekäme er dann Scheine mit einem Wert von vier, fünf oder sechs Leistungspunkten. Wenn der Studierende Münzen und Scheine im Wert von 180 oder 120 Leistungspunkten zusammenhat, würde er diese einfach beim Prüfungsamt gegen einen Bachelor- oder Masterabschluss eintauschen.

Aber natürlich funktioniert dieses Verfahren nicht, weil ja nicht beliebige Leistungspunkte kombiniert werden können. Auf jeder Münze und jedem Schein müssten deshalb Zusatzinformationen vermerkt werden. Es muss genau markiert werden, für welche Leistung eine Münze oder ein Schein erworben wurde, durch wen diese Leistung erbracht wurde und wann diese erworbenen Leistungspunkte eventuell wieder verfallen. Erst wenn jeder dieser 180 oder 120 Leistungspunkte in einem vorher genau festgelegten Zusammenhang erworben wurde, können sie auch gegen einen spezifischen Bachelor oder Master in Politikwissenschaft, Logik oder Pflegekunde eingetauscht werden.

Im System vor Bologna konnte das alles noch händisch erledigt werden. Ein Studierender ließ sich seine in einer Vorlesung oder in einem Seminar erbrachten Leistungen auf einen ,Schein“ von dem Dozenten oder einer Dozentin bestätigen. Die notwendigen Kontextinformationen wie Name des Studierenden, Titel und Art der Veranstaltung, Form der Leistungserbringung und Datum wurden dann einfach auf dem Stück Papier vermerkt und mit einer Unterschrift zertifiziert. Die Studierenden sammelten diese Scheine in ihrem ,Sparstrumpf ${ }^{\star}$ - einer kleinen Mappe für die Scheine. Wenn die Studierenden die für einen Studienabschluss notwendigen 7,8 oder 12 Scheine gesammelt hatten, trugen sie sie zum Prüfungsamt und bekamen dafür - in Kombination mit den Abschlussprüfungen - ihr Zeugnis.

Eine solche ,Low-Tech-Variante" kann aber bei zu kalkulierenden Verrechnungseinheiten von 120, 180 oder 240 Leistungspunkten, bei der Erhebung von zusätzlichen Informationen über die möglichen Modulzuordnungen von Leistungspunkten und den komplexen Einschränkungen von Punktkombinationen nicht mehr funktionieren. Deswegen wurden an den Hochschulen mit der Einführung der Kunstwährung ECTS die bis dahin 
bestenfalls rudimentär vorhandenen IT-Systeme zur Prüfungs- und Veranstaltungsverwaltung massiv ausgebaut. Keine Hochschule scheint heutzutage mehr ohne komplexe Modul-Programm-Planungs-Programme, elektronische Vorlesungsverzeichnisse oder Campus-Management-Systeme auszukommen. Das Sammeln von Scheinen in einem heimischen ,Sparstrumpf" wurde ersetzt durch ein System, in dem jede Leistung nur wahrnehmbar ist, wenn sie in einer ,Studiengangs-Daten-Bank' abgelegt ist.

\section{Die Diffusionskraft einer Kunstwährung}

Kunstwährungen sind keine statischen Verrechnungseinheiten, sie können sich entweder durch Entscheidungen der Währungshüter oder auch durch die Verwendung in der Praxis verändern. Die Vielfliegerprogramme beispielsweise waren ursprünglich relativ einfache Tauschsysteme, bei denen durch Flüge gesammelte Punkte gegen Freiflüge umgetauscht werden konnten. Schrittweise wurden diese Vielfliegerprogramme dann mit zusätzlichen Eigenschaften aufgeladen, sodass die Bonuspunkte auch zusätzlich direkt gegen Geldzahlungen erworben, an andere Personen übertragen und gegen eine Vielzahl von Produkten und Leistungen eingetauscht werden können.

Das Interessante an der ,Kunstwährung' ECTS ist die Art und Weise, wie sie erst über einen Zeitraum von mehreren Jahrzehnten immer mehr mit den Eigenschaften des Speicherns, Sammelns, Transferierens und Tauschens aufgeladen wurde. Ursprünglich wurde ECTS in den späten achtziger Jahren des zwanzigsten Jahrhunderts lediglich dafür entwickelt, die Leistungen von Studierenden aus einigen wenigen europäischen Ländern, die ein oder zwei Semester an einer ausländischen Partneruniversität verbracht haben, mit Leistungen an der Heimatuniversität zu verrechnen. Durch die Einführung der ECTSPunkte brachte eine Studentin bei ihrer Rückkehr dann nicht nur die Information mit, dass sie zwei Seminare belegt, eine mündliche Prüfung abgelegt und eine Hausarbeit geschrieben hat, sondern auch eine Schätzung, wie viel Zeit dafür - nach Kalkulation ihrer Gastuniversität - dafür aufgewendet werden musste. Die Leistungen wurden besser transferierbar. Deswegen auch die ursprüngliche Bezeichnung als European Credit Transfer System.

Erst in den Jahren nach der Bologna-Erklärung zeichnete sich dann $a b$, dass das ECTS-System nicht nur ein Transfersystem einzelner Leistungen sein soll, sondern auch dafür genutzt werden kann, alle Anforderungen in einem Studium in ECTS-Punkten zu beund verrechnen. Durch die Abbildung aller erwarteten Leistungen in Form dieser Zeitwährung wurde es möglich, die Kunstwährung dafür zu nutzen, dass alle Studierenden nicht nur diejenigen, die an mehreren Hochschulen studiert haben -, am Ende ihre Punkte gegen ein definiertes Endprodukt, einen Bachelor- oder Masterabschluss, tauschen konnten. Voraussetzung für diesen Tausch gegen einen Abschluss ist, dass diese ECTS-Punkte auch gesammelt werden können. Im Rahmen des Bologna-Prozesses wurde deswegen von vielen Universitäten die Möglichkeit eingeführt, dass die Studierenden ihre Punkte in den kleinen bei den Prüfungsämtern angesiedelten elektronischen Schließfächern sammeln konnten. Aus einem Transfer-System wurde so zusätzlich immer mehr auch ein AkkumulationsSystem.

Welche Rolle hat die Kunstwährung ECTS jetzt bei dem überraschenden Verbreitungserfolg der Bologna-Reformen gespielt? 


\subsection{Die räumliche Ausbreitung der Kunstwährung}

Als Erstes sticht ins Auge, wie schnell sich der Bologna-Prozess räumlich ausgebreitet hat. Während in der Vorbereitung zur Bologna-Konferenz nur die Bildungsminister Deutschlands, Italiens, Englands und Frankreichs in einer Erklärung die Einführung einer gemeinsamen Kunstwährung anregten, waren es bei der Bologna-Konferenz bereits dreißig europäische Staaten. Nur zehn Jahre nach der Verabschiedung waren es dann schon sechsundvierzig Staaten, die dem Bologna-Prozess beigetreten waren. Und es ist jetzt schon zu beobachten, dass auch Staaten in Asien, Afrika, Amerika oder Australien die Entwicklungen in Europa mit Interesse zur Kenntnis nehmen und als Beobachter an den Konferenzen der Bildungsminister teilnehmen.

Dabei überrascht weniger die Bereitschaft, sich in Erklärungen zu so hehren Werten wie ,Förderung der internationalen Mobilität‘, ,Sicherung von Qualität in der Bildung‘ oder ,Zugang zu Bildung unabhängig von sozialer Herkunft‘ zu bekennen. Schon die Erfahrungen der UNESCO, der UN-Organisation für Erziehung, Wissenschaft und Kultur, zeigt seit ihrer Gründung im Jahr 1945, dass es (fast) allen ungefähr zweihundert Staaten der Weltgesellschaft leichtgefallen ist, sich zu abstrakt formulierten Werten wie Wissenschaftsförderung, Bildungsgerechtigkeit, Gesundheitserziehung, Geschlechtergleichstellung oder Kulturpflege zu bekennen. Der Beitritt zu einem lockeren Bündnis, in dem vorrangig das Bekenntnis zu allgemein akzeptierten Werten gepflegt wird, ist also billig und überrascht deswegen nicht. Überraschender ist in Bezug auf den Bologna-Prozess also vielmehr, wie bereitwillig die Staaten - neben der Zweigliedrigkeit eines berufsqualifizierenden Basisstudiums und eines darauf aufbauenden Masterstudiums - die Kunstwährung ECTS übernommen haben.

Diese Bereitwilligkeit zur Übernahme einer Kunstwährung ist - so mein Argument darauf zurückzuführen, dass in den betreffenden Staaten in der Regel keine eigene Zeitwährung zur Berechnung der Arbeitslast zur Verfügung stand. Es bestand in diesen Staaten bisher keine Verrechnungseinheit, mit der die Zeit der Studierenden für die Vor- und Nachbereitung von Veranstaltungen, für das Absolvieren von Prüfungsleistungen oder das Verfertigen von Hausarbeiten gemessen wurde, sodass es keine Konkurrenz zu der neuen Kunstwährung gab. Der Expansionsprozess der Kunstwährung ist vergleichbar zum Vordringen einer Geldwährung in eine Region, in der bisher lediglich Gütertausch bekannt war. Die Übernahme der neuen Währung verspricht nicht nur, den Vergleich und die Verrechnung mit neuen Tauschpartnern möglich zu machen, sondern auch den Vergleich und die Verrechnung im Inneren zu erleichtern, ohne dass dafür bestehende Berechnungsformen gänzlich aufgegeben werden müssen.

Diese räumliche Expansion der Kunstwährung stößt erst dann an Grenzen, wenn in einem Land bereits eine andere, gut eingeführte Bildungswährung vorhanden ist. Schließlich erscheint es nicht einleuchtend, weswegen man eine Währung übernehmen soll, wenn es doch bereits eine eigene Währung gibt, mit der das Gleiche - vielleicht nur auf andere Art und Weise - berechnet werden muss. Wie bereits die Schwierigkeiten bei der gegenseitigen Verrechnung von an europäischen und US-amerikanischen Universitäten erbrachten Leistungen zeigen, werden dann eigene Modelle zur Verrechnung der Studienleistungen etabliert, die häufig noch nicht einmal Bezug nehmen auf die in den beiden Regionen herrschenden Verrechnungseinheiten. 


\subsection{Die soziale Ausweitung einer Kunstwährung}

Es fällt uns fast nicht mehr auf, dass die vermeintliche Arbeitsbelastung für jeden Studenten, jede Studentin in einem Bologna-Studiengang in der Kunstwährung ECTS berechnet wird. Dabei ging es - wie oben argumentiert - anfangs lediglich um die ,Einführung eines Leistungspunktesystems', um Studierenden, die an mehreren Uniersitäten studieren, die Anrechenbarkeit von Studienleistungen zu ermöglichen. Erst im Laufe des Prozesses bildete sich das Prinzip heraus, das Kreditpunktesystem nicht nur zum Transfer von Leistungen zwischen verschiedenen Universitäten $\mathrm{zu}$ benutzen, sondern es auch verpflichtend für die Studierenden vorzusehen, die ihr gesamtes Studium an einer einzigen Universität absolvieren.

Diese soziale Ausweitung der Kunstwährung überrascht, weil sowohl vor als auch nach dem Anstoßen des Bologna-Prozesses nur eine geringe Anzahl von Studierenden überhaupt während eines Studiums zwischen Universitäten wechselt. Kritische Beobachter vergleichen deswegen die verbindliche Einführung der ECTS-Punkte, die schon bei ihrer Erprobung für die Mobilitätsprogramme der EU in den 1990er Jahren nicht gerade handlich gewesen sind, mit einem Gips, den man nicht nur Studierenden mit einem gebrochenen Bein verordnet, sondern vorsorglich allen.

Aber selbstverständlich wird die Bezeichnung von ECTS-Punkten als Gips für Gesunde von den wenigsten Verfechtern der Bologna-Reform geteilt. Vielmehr wurden zusätzliche Ziele entdeckt, die mit dem eigentlich nur für das Ziel ,Mobilitätsförderung gedachten Mittel ECTS-Punkte erreicht werden können. Plötzlich wurde das Argument gebracht, dass die ECTS-Punkte ja nicht nur für die Verrechnung der Mobilität zwischen Universitäten verschiedener Staaten genutzt werden können, sondern auch für die Verrechnung verschiedener Veranstaltungen zwischen verschiedenen Instituten einer Universität. Schnell war dann auch die Rede davon, dass mit ECTS-Punkten der Zeitaufwand für einen Studiengang genauer bestimmt werden kann und dass durch eine Berechnung des gesamten Studiengangs in dieser Zeitwährung sowohl eine Unter- als auch Überforderung von Studierenden verhindert werden kann.

Diese ,Erfindung‘ von neuen Zielen für ein ursprünglich für ein ganz anderes Ziel eingeführtes Mittel ist in der Organisationsforschung sehr gut untersucht (vgl. Kühl 2011). Hat man, um ein Beispiel aus dem Bereich der Wirtschaft zu nennen, als Weltbank nach dem Zweiten Weltkrieg Know-how für das Management von Projekten zum Wiederaufbau in Europa entwickelt, bietet es sich an, dieses Wissen auch für die Durchführung und Finanzierung von Projekten in sogenannten , unterentwickelten' Ländern in Asien, Afrika und Lateinamerika zu nutzen (vgl. Hanke 1996).

Normalerweise wird aber in der Organisationsforschung davon ausgegangen, dass sich vielfältige Hürden auftun, wenn Mittel, die für ein bestimmtes Ziel entwickelt wurden, für neue Ziele eingesetzt werden sollen. Im Fall des Bologna-Prozesses war diese Ausweitung des Mitteleinsatzes vergleichsweise unproblematisch, weil die Leistungsanforderungen sowieso in einer numerischen Form gefasst sind. Hat man erst einmal ein auf Zeitstunden basierendes Transfersystem etabliert, dann ist es ein vergleichsweise geringer Aufwand, dieses System auch für die Akkumulation von Leistungen im Rahmen eines Studienganges zu nutzen. Wenn man sowieso als Maßnahme der Mobilitätsförderung alle an einer Universität angebotenen Veranstaltungen in eine Kunstwährung umrechnen muss, dann kann man - so die Logik - auch gleich die gesamte Planung von Studiengängen auf dieser Kunstwährung aufbauen. 


\subsection{Die sachliche Ausdehnung einer Kunstwährung}

Ursprünglich war die Kunstwährung ECTS nur zur Berechnung des Arbeitsaufwandes für einzelne Veranstaltungen und Prüfungen gedacht. Hat man aber erst einmal den vermeintlichen Arbeitsaufwand von Studierenden für jede Veranstaltung und jede Prüfung bestimmt, kann man diese Zahl nahezu beliebig mit anderen Zahlen kombinieren.

Diese Aufladung der ECTS-Punkte durch die Kombination mit anderen Zahlen betrifft erst einmal vorrangig die Studierenden. Ein Feld, in dem die Kunstwährung ECTS bereits vielfach mit anderen Zahlen kombiniert wird, ist die Notengebung. Weil ECTS-Punkte nur den durchschnittlichen Arbeitsaufwand von Studierenden messen, müssen getrennt davon die Noten für Arbeitsleistungen erhoben werden. In vielen Universitäten werden die Noten jetzt bereits schematisch nach der Anzahl der Leistungspunkte für ein Arbeitspaket gewichtet. Je mehr Leistungspunkte für ein Modul vergeben werden, desto wichtiger ist die Note für dieses Modul für die Endnote. Aber Universitäten experimentieren jetzt schon damit, die Zulassung zu weiterführenden Studiengängen davon abhängig zu machen, wie viele Leistungspunkte während des Bachelorstudiums in den für den Masterstudiengang relevanten Modulen erworben werden. Eine Zulassung zu einem Studiengang ,Politische Kommunikation“ erhält man dann eben nur, wenn man während des Bachelorstudiums mindestens 60 Leistungspunkte in Politikwissenschaft und 60 Leistungspunkte in Kommunikationswissenschaft erworben hat. Aber auch ist jetzt schon - basierend auf Erfahrungen in den USA - abzusehen, dass auch die Studiengebühren mit erworbenen ECTSPunkten kombiniert werden können. Studierende würden - so die Idee - dann nicht mehr einen Pauschalbetrag pro Semester bezahlen, sondern ihre Gebühren abhängig von den ihren Modulen zugerechneten ECTS-Punkten bezahlen (vgl. zur Debatte in den USA Altbach 2000: 85).

Aber auch die Leistung von Lehrenden kann an die Kunstwährung ECTS gebunden werden. Bisher wird das Deputat der Lehrenden an den meisten Universitäten nach einem relativ einfachen und bürokratiearmen Schema berechnet. Egal ob ein Lehrender eine Professorin, ein Assistent oder eine Lehrbeauftragte ein Seminar, eine Vorlesung, eine Übung oder ein Kolloquium abhält - jede ,Kontaktstunde' mit den Studierenden wurde ihnen als Lehrdeputatsstunde angerechnet. Durch die ECTS-Punkte hat man jetzt jedenfalls theoretisch - die Möglichkeit, die Deputate von Lehrenden völlig neu zu bestimmen. Man kann beispielsweise vor- und nachbereitungsintensive Seminarveranstaltungen, in denen Studierende viele ECTS-Punkte erwerben können, bei der Deputatsberechnung für Lehrende höher gewichten als nur mit wenigen ECTS-Punkten gewichtete Übungen, weil man davon ausgehen könnte, dass diese nach Schema $F$ abgehalten werden und von Lehrenden nicht intensiv vor- und nachbereitet werden müssen. Bisher ist dies lediglich theoretische Gedankenspielerei, aber es ist nur eine Frage der Zeit, bis die eine oder andere hochschulpolitische Denkschmiede anfängt, neben dem European Credit Transfer and Accumulation System auch ein European Teaching Load System (ETLS) zu entwickeln (vgl. für solche ersten Gedankenspiele Yorck 2005).

Aber auch die Leistungsmessung von Instituten, Fakultäten oder auch ganzer Universitäten kann mit der Kunstwährung ECTS verbunden werden. Im Moment scheint es an vielen Universitäten noch eine abstruse Vorstellung, dass die Gesamtzahl an ECTSPunkten nicht nur für einzelne Studenten, sondern auch verallgemeinert für ganze Studiengänge einer Fakultät oder einer Universität erhoben werden. Bisher klingt es für viele Hochschulangehörige kaum vorstellbar, dass ein Institut irgendwann einmal vermeldet, dass im Wintersemester eines Jahres in seinen Veranstaltungen insgesamt 32423 
ECTS-Punkte von Studierenden erworben wurden und Universitäten mit Bildungsministerien Leistungsvereinbarungen abschließen, dass in einem Semester ihre Studierenden mindestens 2,5 Mio. ECTS Punkte erwerben, aber durch die IT-Systeme ließen sich diese Zahlen in vielen Hochschulen jetzt schon innerhalb von wenigen Minuten generieren. Erfahrungen mit dem Kreditpunktesystem in den USA und auch erste Erprobungen in europäischen Staaten deuten darauf hin, dass es wohl nur eine Frage der Zeit ist, bis die Kunstwährung auch in die Gesamtsteuerung von Universitäten über Leistungsindikatoren eingebunden wird.

In der Soziologie wird diese Produktion neuer Zahlen durch die Verkopplung einer Zahl mit anderen Zahlen als „Hyperrealität“ (Vollmer 2006) bezeichnet. Zwar mögen von Beobachtern Zweifel an der Logik bei der Verknüpfung zweier Zahlen - z. B. von ECTSPunkten mit Studiengebühren oder von allen an einer Uni erworbenen ECTS-Punkten mit Mittelzuweisungen - geäußert werden, aber durch die Fassung von bestimmten Phänomen in die Form von Zahlen entsteht eine Sogwirkung, diese Zahlen mit anderen Zahlen in Beziehung zu setzen und zu verrechnen.

\section{$6 \quad$ Zu den Sogwirkungen einer Kunstwährung}

Globale Vereinheitlichungen werden häufig als eine Durchsetzung von Standards von oben nach unten beschrieben. Es wird dabei analysiert, wie sich die Entscheidungen von internationalen Organisationen oder internationalen Konferenzen über nationalstaatliche Ratifizierungen bis auf die lokale Ebene durchsetzen (für den in diese Richtung argumentierenden regulationstheoretischen Strang vgl. z. B. Hirsch 2005). Oder es wird davon ausgegangen, dass sich weltweit Ideologien in der Form von nahezu quasireligiösen Rationalitätsannahmen über die Funktionsweise von Wirtschaft, Politik, Recht oder Erziehung ausbilden, die dann einen unmittelbaren Anpassungsdruck auf lokale Praktiken ausüben (für den neoinstitutionalistischen Argumentationsstrang vgl. z. B. Strang/Meyer 1993).

Der durch die Klammer des Bologna-Prozesses gehaltene Reformprozess scheint sich aber eher von ,unten` zu bilden. Der zentrale Faktor für die Entstehung und Durchsetzung von Reformmustern scheint dabei in den Experimenten mit der Kunstwährung ECTS zu liegen. Es bildeten sich so dabei immer mehr die Konturen einer Kunstwährung heraus, die mit Eigenschaften des Transfers, Sammelns, Speicherns und Tauschens aufgeladen wurde.

Besonders unter Planungsgesichtspunkten erhielt die Kunstwährung ECTS gerade aus der Perspektive von Ministerien und Hochschulleitungen eine hohe Attraktivität, weil plötzlich viele vorher eher im Dunkeln von Seminaren, Vorlesungen und Studierstuben ablaufenden Prozesse wenigstens von ihrem Zeitaufwand her berechen-, kontrollier- und planbar erschienen ${ }^{17}$. Aber diese Attraktivität für die Leitungsebene ist - so das Argument dieses Artikels - nicht der Grund für den Durchsetzungserfolg der ECTS-Punkte gewesen: Vielmehr entwickelte das Punktesystem eine sich weitgehend einer zentralen Planung, der sich die Hochschulen im ,Bologna-Raum“ in der einen oder anderen Form unterwerfen müssen, entziehende Eigendynamik. Man kann hier von der Sogwirkung der Verrechnungs-

\footnotetext{
${ }^{17}$ Das Ergebnis war jedoch eine bis dahin nicht gekannte Komplexitätssteigerung in der Konzeption und Durchführung von Studiengängen an den Universitäten, die sich am besten mit dem Begriff des „Sudoku-Effekts“ fassen lässt (Kühl 2010). Zu den ungewollten Nebenfolgen der Hochschulreform siehe ausführlich Kühl 2012.
} 
einheiten reden, die bei aller Heterogenität in den Details Entwicklungslinien auf einen gemeinsamen quantitativen Nenner bringen.

\section{Literatur}

Adelman, C. (2009): The Bologna Process for U.S. Eyes: Re-learning Higher Education in the Age of Convergence. Washington, DC.

Altbach, P. (2000): Die Messung von Lehr- und Lernleistungen: Credit-Systeme an US-amerikanischen Hochschulen. In: Schwarz, S./Teichler, U. (Hg.): Credits an deutschen Hochschulen: Kleine Einheiten - große Wirkung. Neuwied, Kriftel: 79-89.

Berliner Erklärung (2003): Den Europäischen Hochschulraum verwirklichen. Kommuniqué der Konferenz der europäischen Hochschulministerinnen und -minister am 19.9.2003 in Berlin.

BLK (2002): Modularisierung in Hochschulen. Handreichung zur Modularisierung und Einführung von Bachelor- und Masterstudiengängen. Bonn: Bund-Länder-Kommission für Bildungsplanung und Forschungsförderung.

Bloor, D. (1994): What Can the Sociologist of Knowledge Say About 2+2=4? In: Ernest, P. (ed.): Mathematics, Education and Philosophy: An International Perspective. London: 21-32.

Boli, J./Lechner, F. (2001): Globalization and World Culture. In: Smelser, N. J./Baltes, P. B. (eds.): International Encyclopedia of the Social and Behavioral Sciences. Amsterdam: 6261-6266.

Bologna Follow-Up Group Deutschland (2007) Bericht der Bologna Follow-Up Group. Bonn.

Booysen, F. (2002): An Overview and Evaluation of Composite Indices of Development. Social Indicators Research, 59:115-151.

Erhardt, M. (2000): Einleitung. In: Stifterverband für die Deutsche Wissenschaft (Hg.): Credits an deutschen Hochschulen. Transparenz - Koordination - Kompatibilität. Bonn: 3-4.

European Communities (2009): ECTS Users' Guide. Luxemburg: Office for Official Publications of the European Communities.

Gehmlich, V. (2000): Möglichkeiten und Grenzen des European Credit Transfer Systems (ECTS). In: Schwarz, S./Teichler, U. (Hg.): Credits an deutschen Hochschulen: Kleine Einheiten - große Wirkung. Neuwied, Kriftel: 57-76.

Hanke, S. (1996): Weiß die Weltbank, was sie tut? Über den Umgang mit Unsicherheit in einer Organisation der Entwicklungsfinanzierung. Soziale Systeme, 2: 331-360.

Heintz, B. (2007): Zahlen, Wissen, Objektivität: Wissenschaftssoziologische Perspektiven. In: Mennicken, Andrea; Hendrik Vollmer (Hg.): Zahlenwerk. Kalkulation, Organisation und Gesellschaft. Wiesbaden: 65-86.

Heintz, B. (2010): Numerische Differenz. Überlegungen zu einer Soziologie des (quantitativen) Vergleichs. Zeitschrift für Soziologie, 39: 162-181.

Hirsch, J. (2005): Materialistische Staatstheorie. Transformationsprozesse des kapitalistischen Staatensystems. Hamburg.

Keller, A. (2005): Reise nach Bologna - Holzweg oder Königsweg? Risiken des Europäischen Hochschulraums. In: Gützkow, F./Quaißer, G. (Hg.): Jahrbuch Hochschule gestalten 2005. Denkanstöße zum Bologna-Prozess. Bielefeld: 63-76.

KMK (2004): Rahmenvorgaben für die Einführung von Leistungspunktsystemen und die Modularisierung von Studiengängen. o.O.: Beschluss der Kultusministerkonferenz vom 15.9.2000 i.d.F. vom 22.10.2004.

Kühl, S. (2010): Der Sudoku-Effekt. Zu den Gründen und Folgen der Komplexitätssteigerung an den Hochschulen. Soziale Systeme, 16: 444-460.

Kühl, S. ( 2012): Der Sudoku-Effekt. Die Hochschulen im Teufelskreis der Bürokratie. Bielefeld.

Liessmann, K. P. (2008): Theorie der Unbildung. Die Irrtümer der Wissensgesellschaft. München.

Maeße, J. (2010): Die vielen Stimmen des Bologna-Prozesses. Zur diskursiven Logik eines bildungspolitischen Programms. Bielefeld.

Marx, K. (1953): Die Frühschriften. Stuttgart.

Pasternack, P. (2000): Die Hochschulqualitätsdebatte. Hochschulwesen, 2: 38-43. 
Pentland, B. T. (1993): Grammatical models of organizational processes. Los Angeles, Calf: Anderson Graduate School of Management, University of California, Los Angeles.

Radcliffe, V. (1999): Knowing Efficiency: The Enactment of Efficiency in Efficiency Auditing. In: Accounting, Organizations and Society, 24: 333-362.

Roscher, F. (2000): Das operative Regelwerk von Credit-Systemen. In: Schwarz, S./Teichler, U. (Hg.): Credits an deutschen Hochschulen: Kleine Einheiten große Wirkung. Neuwied, Kriftel: 45-56.

Rottenburg, R.(2001): Kultur der Entwicklungszusammenarbeit mit Afrika. In: Wippel, S./Cornelssen, I. (Hg.): Entwicklungspolitische Perspektiven im Kontext wachsender Komplexität. Festschrift für Prof. Dr. Dieter Weiß. Bonn: 349-377.

Schwarz, S./Rehburg, M. (2003): Weit mehr als eine Studienreform die Potenziale von Credit-Systemen und gestuften Studiengängen an deutschen Hochschulen. In: Schwarz, S./Teichler, U. (Hg.): Universität auf dem Prüfstand. Konzepte und Befunde der Hochschulforschung. Frankfurt a.M., New York: 137-156.

Strang, D./Meyer, J. W. (1993): Institutional Conditions for Diffusions. Theory and Society, 22: 487-511.

Vollmer, H. (2006): How to Do More With Numbers. Elementary Stakes, Framing, Keying, and the Three-dimensional Character of Numerical Signs. Bielefeld: unveröff. Ms.

Wagner, W. (2007): Uni-Angst und Uni-Bluff heute. Wie studieren und sich nicht verlieren. Berlin.

Weizsäcker, E. von (1970): Baukasten gegen Systemzwänge. München.

Winter, M. (2009): Das neue Studieren. Chancen, Risiken, Nebenwirkungen der Studienstrukturreform: Zwischenbilanz zum Bologna-Prozess in Deutschland. Wittenberg.

Yorck, H. (2005): „Teaching Points“ als neue Planungsgröße für die Lehre? Ein Vorschlag des Centrums für Hochschulentwicklung. Forschung \& Lehre, 11: 600-610. 